\title{
Validation of a Two-Antenna Reverberation-Chamber Technique for Estimating the Total and Radiation Efficiency of Antennas
}

\author{
Christopher L. Holloway ${ }^{1}$, Richard S. Smith ${ }^{2}$, Colton R. Dunlap ${ }^{1}$, Ryan J. Pirkl ${ }^{1}$, John Ladbury ${ }^{1}$, \\ William F. Young ${ }^{1}$, David A. Hill ${ }^{1}$, William R. Hansell ${ }^{2}$, Marc A. Shadish ${ }^{2}$, Kahle B. Sullivan ${ }^{2}$ \\ ${ }^{1}$ National Institute of Standards and Technology (NIST), Electromagnetics Division \\ 325 Broadway, Boulder, CO 80305, USA, 303-497-6184: holloway@boulder.nist.gov \\ ${ }^{2}$ ITT Exelis, 100 Riverside Parkway, Suite 119, Frederickburg, VA 22406
}

\begin{abstract}
Reverberation chambers (RC) are becoming a popular alternative testing facility for a wide range of electromagnetic applications. In particular, these chambers are ideally suited for performing radiated power measurements of either an antenna or a device under test, and as such, it is possible to estimate the efficiency of antennas. In previous work we presented a two-antenna approach that allows for estimating the efficiency of two antennas under test without the need for a reference antenna, nor the need to assume that both antennas are identical. In this paper, we validate this twoantenna approach by showing comparisons with other methods (i.e., comparing measurements made in different test facilities and comparisons to other techniques, including numerical calculations) for various antennas.
\end{abstract}

\section{Introduction}

A reverberation chamber ( $\mathrm{RC})$ is basically a shielded room (metallic walls) with a complex-shaped metallic rotating paddle (stirrer or tuner) [1]. The paddle is designed to be non-symmetric and is used to create a continuously changing boundary condition of the electromagnetic fields in the chamber. Rotating the paddle creates a well defined statistical environment in a RC, and this statistical environment offers a unique test facility. Electromagnetic RCs are becoming popular as alternative test facilities for both electromagnetic and electromagnetic compatibility measurements. RCs were initially used in acoustics for various applications [2], but then later emerged in the electromagnetic community, where they were first used as high-amplitude field test facilities for electromagnetic interference and compatibility. RCs are currently used for a wide range of other measurement applications: (1) radiated immunity of components and large systems, (2) radiated emissions, (3) shielding characterizations of cables, connectors, and materials, (4) antenna measurements (including

U.S. government work, not protected by U.S. copyright efficiency), (5) probe calibration, (6) characterization of material properties, (7) absorption and heating properties of materials, (8) biological and biomedical effects, (9) tests of wireless devices, and (10) simulations of various wireless multipath environments (see [1] and [3] for a list of papers covering the different applications).

RCs are ideally suited for performing radiated power measurements of either an antenna or device under test, and as such, it is possible to estimate the efficiency of antennas. Consider two antennas placed inside a reverberation chamber; see Fig. 1. Antenna $A$ is connected to port 1 of a vector network analyzer (VNA) via a cable running through the bulkhead of the chamber, and antenna $B$ is similarly connected to port 2 of the VNA. Antenna $A$ is used to couple energy into the chamber and is the transmitting antenna, and antenna $B$ is the receiving antenna.

In both [4] and [5] it is shown that the product of the two antenna efficiencies is given by

$$
\eta_{A}^{\text {total }} \eta_{B}^{\text {total }}=\frac{16 \pi^{2} V}{\lambda^{3} \omega}\left\langle\left|S_{21}\right|^{2}\right\rangle \frac{1}{\tau_{R C}}
$$

where $\eta_{A}^{\text {total }}$ and $\eta_{B}^{\text {total }}$ are the total efficiencies (including both radiation and mismatch) of the two antennas, $\mathrm{V}$ is the volume of the RC, $\lambda$ is the freespace wavelength, \langle\rangle represents an ensemble average, and $\tau_{R C}$ is the chamber decay period (see [5][7] for a discussion on determining $\tau_{R C}$ from frequency-domain $S$-parameter data).

Alternatively, as discussed in [5] the efficiency of one antenna can be obtained if we know the efficiency of a reference antenna, using

$$
\eta_{A}^{\text {total }}=\frac{P_{A U T}}{P_{r e f}} \eta_{r e f},
$$

where $\eta_{r e f}$ is the efficiency of the reference antenna, $P_{A U T}$ is the power at the receive antenna $\left(\mathrm{A}_{\mathrm{RX}}\right)$ for the 
antenna under test (AUT), and $P_{\text {ref }}$ is the power at the receiving antenna for the reference antenna $\left(\mathrm{A}_{\text {ref }}\right)$; see Fig. 2 for a diagram of these terms.

The problem with the formulation given in (1) is that in order to determine the efficiency one must assume the two antennas are identical, while the problem with the formulation given in (2) is that the efficiency of a reference antenna is required. In a recent publication we presented three different approaches for determining both the radiation and total efficiencies of unknown antennas that overcome these limitations and assumptions. We presented a one-antenna approach, a two-antenna approach, and a threeantenna approach (see [5], [8], and [9] for details). In this paper, we validate the two-antenna approach by showing comparisons with other methods for four different antennas.

\section{Two-Antenna Approach}

Consider one of the antennas placed in the chamber, as depicted in Fig. 3. The details of the derivation for the two-antenna approach are given in [5], and only highlights are given here. However, some of the expressions are written in different forms here. In [5] it is shown that the total efficiency of antenna $A$ is given by:

$$
\left(\eta_{A}^{\text {total }}\right)^{2}=\left\langle\left|S_{11}\right|^{2}\right\rangle \frac{P_{T X, A}}{\left\langle P_{r f, A}\right\rangle},
$$

where $P_{T X, A}$ is the transmitted power in the chamber for antenna A. Once this energy gets into the chamber, a portion is dissipated in the chamber walls and paddle $\left(P_{R C}\right)$ and a portion of the energy reflects off the chamber walls and paddle and is captured by the antenna (this power is referred to as $P_{r f, A}$ ).

For two antennas placed inside the chamber (see Fig. 3), Hill [1] has shown that $P_{T X, A}$ is related to the quality factor of the chamber $(Q)$ and the power received by a second antenna $\left(P_{R X, A}\right)$ by the following

$$
P_{T X, A}=\left\langle P_{R X, A}\right\rangle \frac{16 \pi^{2} V}{Q \lambda^{3}} .
$$

Substitute this into (3) to obtain

$$
\left(\eta_{A}^{\text {total }}\right)^{2}=\frac{16 \pi^{2} V}{Q \lambda^{3}}\left\langle\left|S_{11}\right|^{2}\right\rangle \frac{\left\langle P_{R X, A}\right\rangle}{\left\langle P_{r f, A}\right\rangle} .
$$

In [1] and [10], Hill and Ladbury show that if we assume the RC is a so-called "ideal" performing chamber (which implies the chamber is well stirred with a large number of modes and a large modedensity) the last term in this expression can be approximated as

$$
\frac{\left\langle P_{r f, A}\right\rangle}{\left\langle P_{R X, A}\right\rangle}=e_{b},
$$

where $e_{b}$ is referred to as the backscattered coefficient. For an ideal chamber, [1] and [5] show that:

$$
\left.e_{b}\right|_{\text {ideal }}=2 \text {. }
$$

This result is analogous to the enhanced backscatter that has been derived for scattering by a random medium. The physical mechanism for this is the coherent addition of rays in the backscatter direction [1] and [10], and is the same in both reverberation chambers and in scattering by random media. This ratio equals 2 for an ideal chamber. However, due to imperfections in the chamber performance this ratio may only approach " 2 ". In fact, this ratio can be thought of as a quality measure for the chamber performance; the closer it is to a value of 2 , the better performing the chamber is (i.e., a well-stirred chamber with a large mode-density). This ratio serves as a means to assess the imperfections of a chamber as discussed in [11].

With (6) and (7), the efficiency of antenna A is given by

$$
\left(\eta_{A}^{\text {total }}\right)^{2}=\frac{16 \pi^{2} V}{Q \lambda^{3}}\left\langle\left|S_{11}\right|^{2}\right\rangle \frac{1}{e_{b}} .
$$

Following a similar process, the efficiency of antenna $\mathrm{B}$ is given by:

$$
\left(\eta_{B}^{\text {total }}\right)^{2}=\frac{16 \pi^{2} V}{Q \lambda^{3}}\left\langle\left|S_{22}\right|^{2}\right\rangle \frac{1}{e_{b}} .
$$

Combining (1), (9) and (10) gives

$$
\begin{aligned}
& \frac{\left\langle\left|S_{11}\right|^{2}\right\rangle}{\left\langle\left|S_{21}\right|^{2}\right\rangle}=e_{b} \frac{\eta_{A}^{\text {total }}}{\eta_{B}^{\text {total }}} \\
& \frac{\left\langle\left|S_{22}\right|^{2}\right\rangle}{\left\langle\left|S_{21}\right|^{2}\right\rangle}=e_{b} \frac{\eta_{B}^{\text {total }}}{\eta_{A}^{\text {total }}}
\end{aligned}
$$

where we used the fact that [1] 


$$
Q=\omega \tau_{R C}
$$

We have two equations and two unknowns (the value for $e_{b}$ and the ratio $\eta_{A}^{\text {total }} / \eta_{B}^{\text {total }}$ ). The ratio of the unknown efficiencies can be eliminated to obtain an expression for $e_{b}$; see [5] and [11]:

$$
e_{b}=\frac{\sqrt{\left\langle\left. S_{11}\right|^{2}\right\rangle\left\langle\left|S_{22}\right|^{2}\right\rangle}}{\left\langle\left|S_{21}\right|^{2}\right\rangle} \text {. }
$$

If the chamber is performing ideally, then $e_{b}=2$. On the other hand, when $e_{b} \neq 2$, the backscatter coefficient $e_{b}$ can be used to assess the performance of the chamber; see [11].

Using $e_{b}$ given in (13) in the expressions in (11), it can be shown that the total efficiencies of the two antennas are

$$
\begin{aligned}
\eta_{A}^{\text {total }} & =\sqrt{\frac{16 \pi^{2} V}{\omega \lambda^{3}} \frac{1}{e_{b}} \frac{\left\langle\left|S_{11}\right|^{2}\right\rangle}{\tau_{R C}}} \\
\eta_{B}^{\text {total }} & =\sqrt{\frac{16 \pi^{2} V}{\omega \lambda^{3}} \frac{1}{e_{b}} \frac{\left\langle\left|S_{22}\right|^{2}\right\rangle}{\tau_{R C}}}
\end{aligned}
$$

The efficiency in (14) is the total efficiency, including mismatch and radiation loss (or ohmic and/or dielectric losses). The radiation efficiency can be obtained by correcting the measured $S_{11}$ and $S_{22}$ to account for the antenna mismatch. Using a similar procedure as given in [12] and [13] for correcting mismatch in $S_{21}$, the radiation efficiencies of the antennas are given by

$$
\begin{aligned}
& \eta_{A}^{\text {rad }}=\sqrt{\frac{16 \pi^{2} V}{\omega \lambda^{3}} \frac{1}{e_{b}} \frac{1}{\tau_{R C}} \frac{\left\langle\left|S_{11}\right|^{2}\right\rangle}{\left(1-\left|\left\langle S_{11}\right\rangle\right|^{2}\right)\left(1-\left|\left\langle S_{11}\right\rangle\right|^{2}\right)}} \\
& \eta_{B}^{\text {rad }}=\sqrt{\frac{16 \pi^{2} V}{\omega \lambda^{3}} \frac{1}{e_{b}} \frac{1}{\tau_{R C}} \frac{\left\langle\left|S_{22}\right|^{2}\right\rangle}{\left(1-\left|\left\langle S_{22}\right\rangle\right|^{2}\right)\left(1-\left|\left\langle S_{22}\right\rangle\right|^{2}\right)}} .
\end{aligned}
$$

\section{Validation of Two-Antenna Technique}

In this section, we show experimental values of $\eta^{\text {total }}$ and $\eta^{\text {rad }}$ obtained from (14) and (15) for various antennas, and compare these estimated efficiencies to values obtained from other approaches. The measurements were taken for different antennas placed in NIST's RC with dimensions of $2.9 \mathrm{~m}$ by $4.2 \mathrm{~m}$ by $3.6 \mathrm{~m}$, see Fig. 4. The experimental set-up was similar to that shown in Fig. 3, in which a VNA was connected to the two antennas under test. We also show measurements performed in ITT Exelis' RC with dimensions of $3.1 \mathrm{~m}$ by $4.8 \mathrm{~m}$ by $3.6 \mathrm{~m}$, as well as measurements performed in a fully anechoic chamber.

The first antenna is a microstrip patch antenna. The antenna was measured in the ITT RC, and the total efficiency obtained from (14) is shown in Fig. 5. The antenna was also measured in a fully anechoic chamber, and the results are also shown in Fig. 5. The fully-anechoic chamber results require a scan and pattern integration to obtain the antenna's total efficiency. Finally, we used a commercial finiteelement program to numerically estimate the total efficiency of the patch antenna. These results are also shown in Fig. 5. All three of these different estimates (RC, anechoic chamber, and numerical calculations) of the total efficiency correlate to one another (within the measurement uncertainties of the RC chamber approach, see [5]).

The second antenna is a dual-ridge horn antenna (silver in color and referred to as the silver horn antenna) with aperture dimensions of $13.5 \mathrm{~cm}$ by $24.5 \mathrm{~cm}$. This antenna was measured two different times (six-months apart) in the NIST chamber, and the radiation efficiency was obtained from (15) for these two measurements. These measurements are shown in Fig. 6. We see that the measurements are very similar (even capturing the broad dip around $3 \mathrm{GHz}$ ), and are within the uncertainties discussed in [5]. The manufacturer of this antenna performed a numerically calculation of the antenna efficiency, in which the actual material properties (in this case aluminum) of the antenna were used in the simulation. Their calculations are also shown in Fig. 6. These numerical results are consistent with the two measurements.

The third antenna is a dual-ridge horn antenna (red in color and referred to as the red horn antenna) with aperture dimensions of $14.4 \mathrm{~cm}$ by $17.6 \mathrm{~cm}$. This antenna was measured in three different test facilities (NIST's RC, ITT's RC, and a fully-anechoic chamber). The two RCs used expression (14) to 
obtain the total efficiency, while the fully-anechoic chamber required a scan and pattern integration to obtain the antenna's total efficiency. The total efficiency for this antenna for the three different facilities is shown in Fig. 7. We see that the measurements are very similar, and are within the uncertainties discussed in [5]. There is more variability in the fully anechoic results, and we believe this is due to a calibration issue in the anechoic chamber.

The fourth antenna is a log-periodic antenna, approximately $1 \mathrm{~m}$ in length. This antenna was measured in three different test facilities: NIST's RC, ITT's RC, and a fully anechoic chamber. The two RC results used the expression given in (15) to obtain the total efficiency, while the fully anechoic chamber required a scan and pattern integration to obtain the antenna's total efficiency. The total efficiency for this antenna for the three different facilities is shown in Fig. 8. We see that the RC results are virtually indistinguishable, and are well within the uncertainties discussed in [5]. Again, there is more variability in the fully anechoic results, and it is believed this is due to a calibration issue in the anechoic chamber. This is seen by the fact that the anechoic-chamber results estimate an efficiency greater than one at one frequency. This issue is currently being investigated.

\section{Summary}

In this paper, we discuss a two-antenna technique for determining the total and radiation efficiency of a single antenna. This technique overcomes the assumptions and limitations needed for the commonly used RC approach. We have validated this approach by making comparisons of various antennas. This validation included comparing measurements made in different test facilities and comparisons to numerical calculations. These comparisons show very good correlation between different RCs and numerical calculations, and to fully-anechoic results. This twoantenna approach has been extended to a one-antenna and three-antenna approach (for details see [5], [8] and [9]). Future work will include a study to fully understand the advantages and disadvantages of all three RC antenna efficiency techniques, as well as a full understanding of the uncertainties in the various approaches.

\section{REFERENCES}

[1] D.A. Hill, Electromagnetic Fields in Cavities: Deterministic and Statistical Theories. N.Y., NY., IEEE Press, 2009.

[2] P.M. Morse and R.H. Bolt, "Sound waves in rooms," Reviews of Modern Physics, vol. 16, no. 2, pp. 69-150, April, 1944.

[3] C.L. Holloway, D.A. Hill, J.M. Ladbury, P.Wilson, G. Koepke, and J. Coder, "On the use of reverberation chambers to simulate a controllable rician radio environment for the testing of wireless devices," IEEE Trans. on Antennas and Propag., , vol. 54, no. 11, pp. 3167-3177, November 2006.

[4] H.G. Krauthauser and M. Herbrid, "Yet another antenna efficiency measurement method in reverberation chambers," IEEE 2010 International Symp. on EMC, July 25-20, Fort Lauderdale, FL, 2010.

[5] C.L. Holloway, H. Shah, R.J. Pirkl, W. Young, J.M. Ladbury, and D.A. Hill, "Reverberation chamber techniques for determining the radiation and total efficiency of antennas," IEEE Trans. on Antennas and Propag., vol. 60, no. 4, April 2012, pp. 1758-1770.

[6] E. Genender, C.L. Holloway, K.A. Remley, J.M. Ladbury, and G. Koepke, 'Simulating the multipath channel with a reverberation chamber: application to bit error rate measurements," IEEE Transactions on EMC, vol. 52, no. 4, pp. 766-777, Nov. 2010.

[7] C.L. Holloway, H.A. Shah, R.J. Pirkl, K.A. Remley, D.A. Hill, J. Ladbury, "Early-time behavior in reverberation chambers and its effect on the relationships between coherence bandwidth, chamber decay time, RMS delay spread, and the chamber build-up time", IEEE Transactions on EMC, accepted and available on IEEE Xplore early access, 2012.

[8] C.L. Holloway, H.A. Shah, R.J. Pirkl, D.A. Hill, and J.M. Ladbury, "A three-antenna technique for determining the efficiency of antennas" in Proc. of 2011 33rd Symposium of Antenna Measurement Techniques Association, Denver, CO, October 16-21, 2011.

[9] C.L. Holloway, R. Smith, C. Dunlap, R. Pirkl, J. Ladbury, and W. Young, "Validation of a one antenna reverberation chamber technique for estimating the total and radiation efficiency of an antenna", Proc of the 2012 IEEE EMC Symposium, Pittsburg, PA, 2012. 
[10] J.M. Ladbury, D.A. Hill, "Enhanced backscatter in a reverberation chamber: inside every complex problem is a simple solution struggling to get out", Proc. in the 2007 IEEE International Symposium on Electromagnetic Compatibility, 9-13 July 2007, pp. 1-5.

[11] C. Dunlap, C.L. Holloway, R. Pirkl, J.M. Ladbury, D.A. Hill, E.F. Kuester, "Characterizing reverberation chambers with measurements of the enhanced backscattered coefficient," Proc. in the 2012 IEEE International Symposium on Electromagnetic Compatibility, Aug. 5-10, 2012, submitted.

[12] J.M. Ladbury, G. Koepke, and D. Camell, "Evaluation of the NASA Langley research center mode-stirred chamber facility," NIST Tech. Note 1508, US Department of Commerce, Boulder, CO, Jan. 1999.

[13] J.M. Ladbury and D.A. Hill, "An improved model for antennas in reverberation chambers", Proc. in the 2010 IEEE International Symposium on Electromagnetic Compatibility, 25-30 July 2010, pp. 663-667.

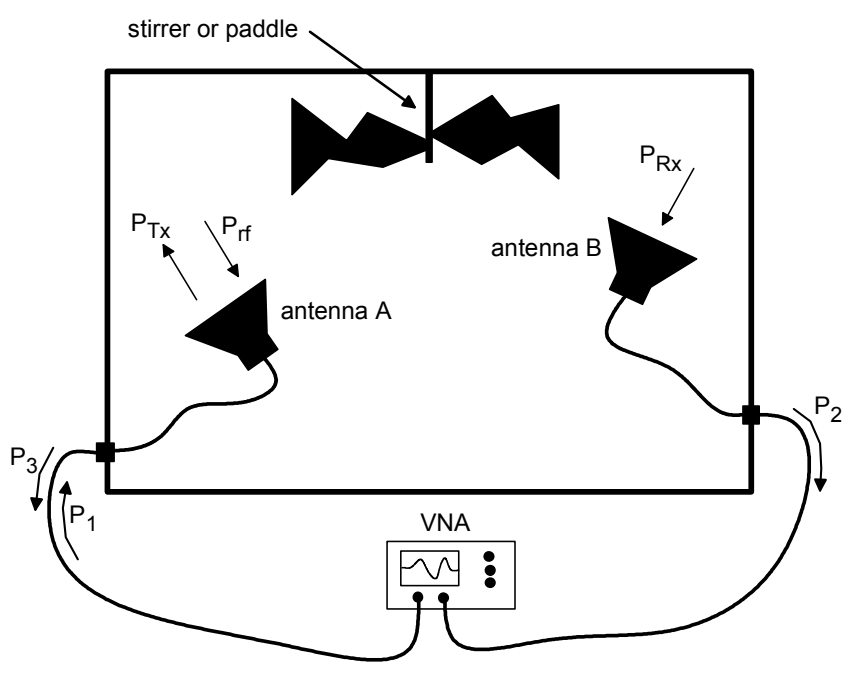

Fig. 1. RC set-up for a two-antenna test.

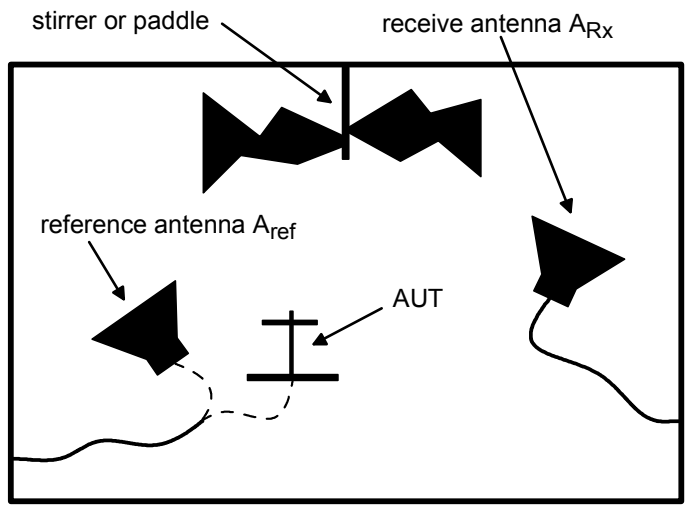

Fig. 2. RC set-up for a reference antenna test.

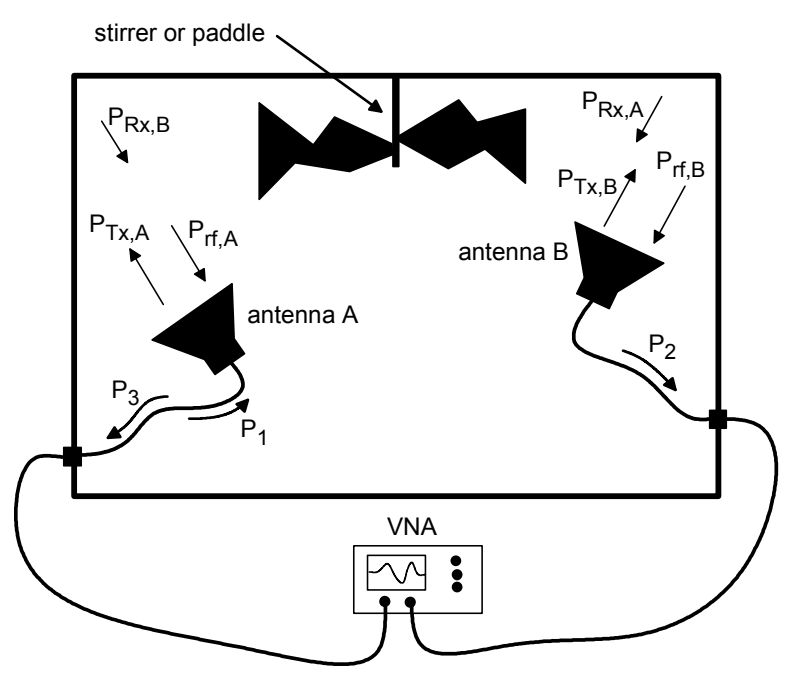

Fig. 3. RC set-up for the two-antenna measurement method for estimating the antenna efficiency.

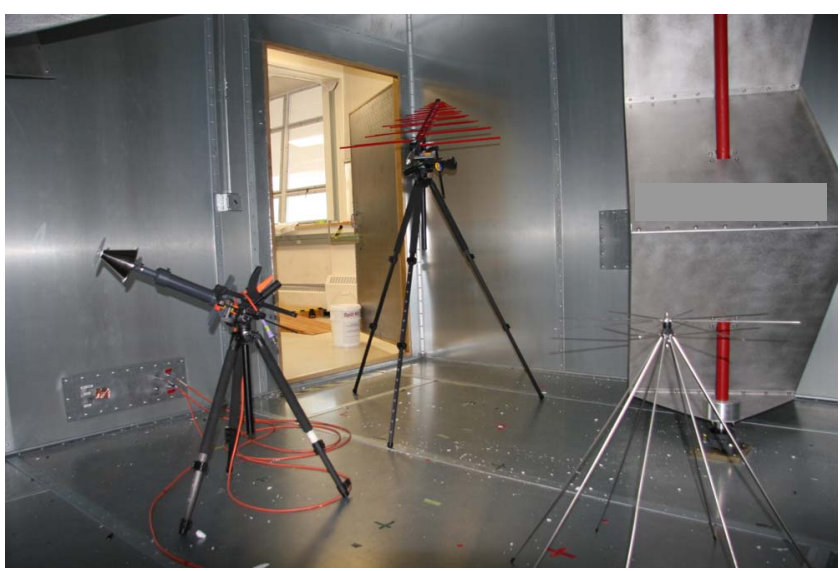

Fig. 4. The NIST RC with antennas placed in the chamber. 


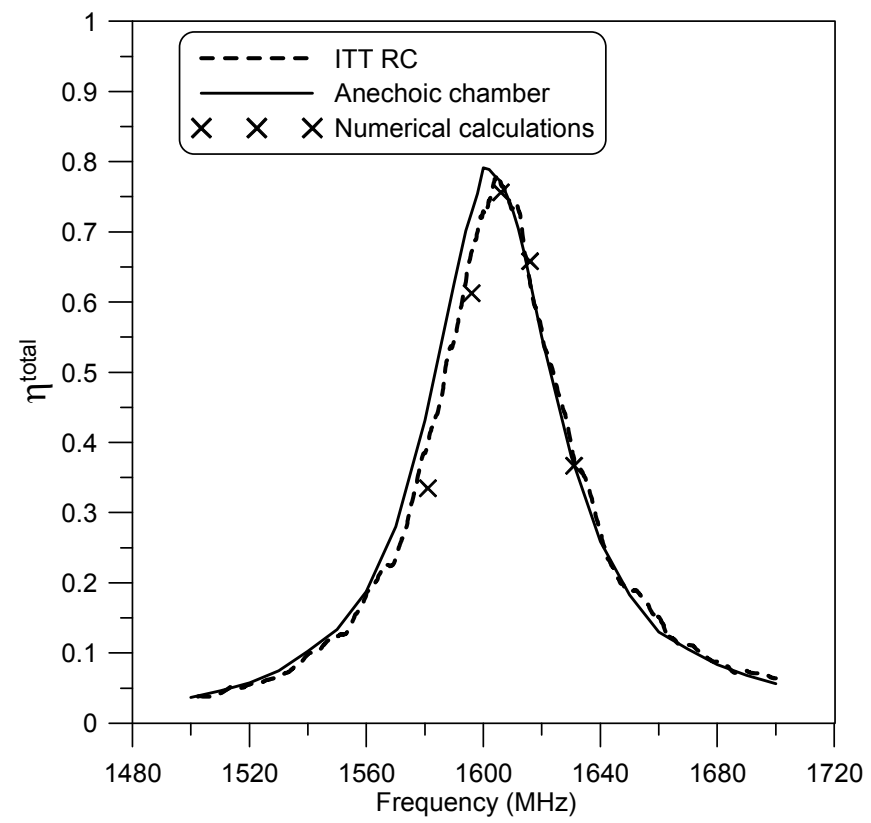

Fig. 5. Microstrip patch antenna efficiency compared to numerical calculations for two different test facilities.

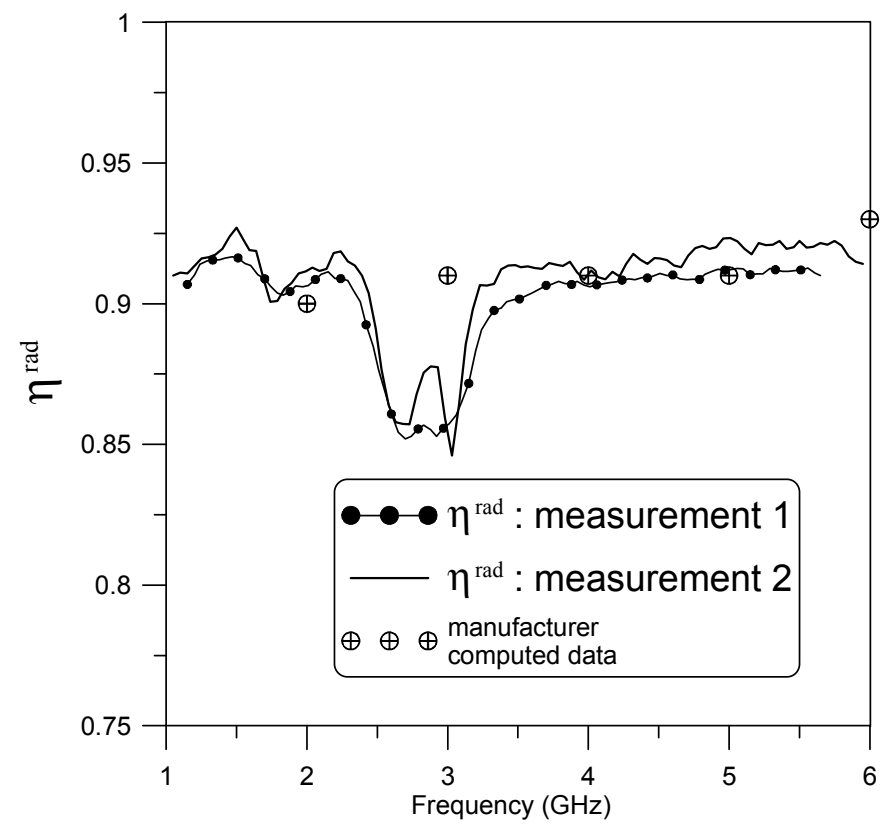

Fig. 6. Silver horn antenna efficiency compared to numerical calculations for two different experiment sets.

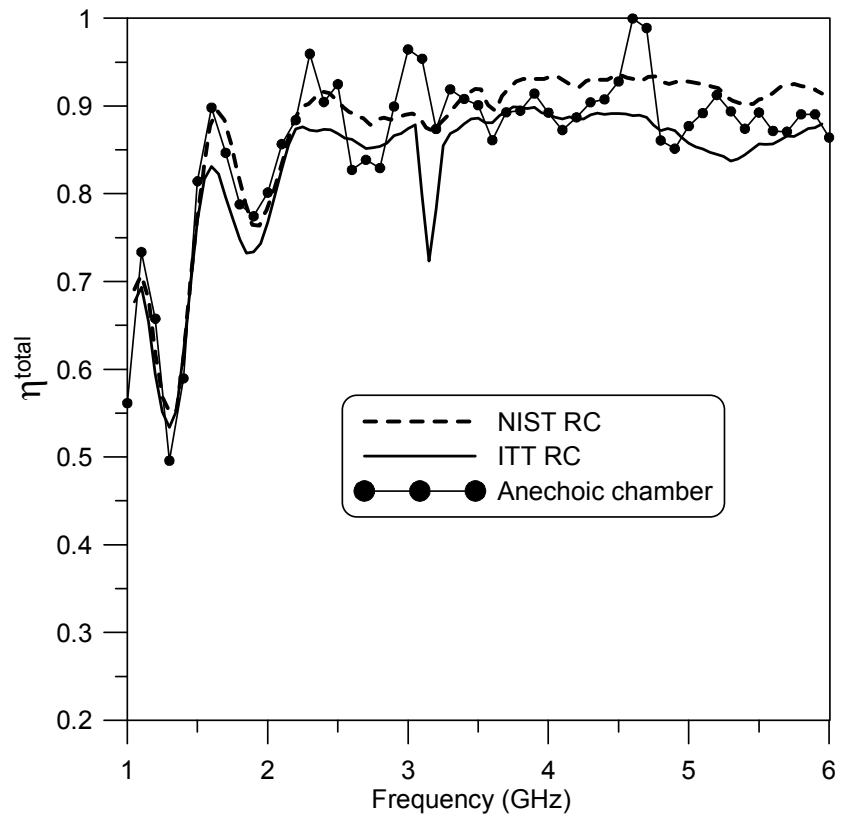

Fig. 7. Red horn antenna efficiency compared for three different test facilities.

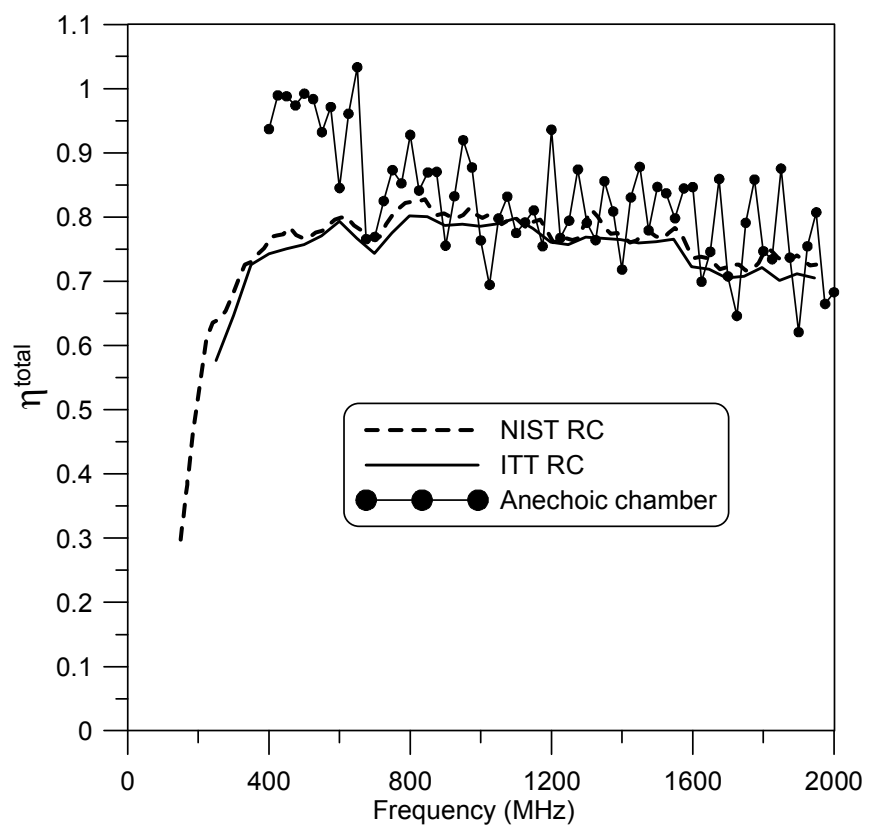

Fig. 8. Log-periodic antenna efficiency compared for three different test facilities. 